\title{
Development of Polymer Resins using Neural Networks
}

\author{
Fabiano A. N. Fernandes, Liliane M. F. Lona \\ Faculdade de Engenharia Química, UNICAMP
}

Abstract: The development of polymer resins can benefit from the application of neural networks, using its great ability to correlate inputs and outputs. In this work we have developed a procedure that uses neural networks to correlate the end-user properties of a polymer with the polymerization reactor's operational condition that will produce that desired polymer. This procedure is aimed at speeding up the development of new resins and help finding the appropriate operational conditions to produce a given polymer resin; reducing experimentation, pilot plant tests and therefore time and money spent on development. The procedure shown in this paper can predict the reactor's operational condition with an error lower than 5\%.

Keywords: Neural networks, polymers, polymerization reactor, simulation.

\section{Introdution}

Development of new polymer resins and new grades of these resins has been one of the great concerns of the polymer industry. Several steps are required to come with new grades produced to satisfy client's needs. First of all, it is necessary to know the end properties of the polymer that the client wants. Second, it is necessary to study which basic polymer physicochemical properties will give these end properties the client wants. And finally, it is necessary to study how to set up the reactor's operational conditions in order to produce that specific polymer.

Great part of the polymer industries produces only a few grades of resins, each one with different physicochemical properties that are suitable for some applications of the plastic industry. The problem is that these resins may not be suitable for some applications of some new developed ones. The plastic processing industry may require a polymer with characteristics that the polymer industry does not produce.

What should be done in this case? The polymer industry should develop a new polymer grade or should the plastic industry be satisfied with a polymer grade that has properties close to the one required for its product but which will not fulfill its quality standards?

With the present level of competition among the polymer industry, a good answer for that question is to develop a new polymer grade for that client.
Specially if the referred amount of polymer to be produced is considerable.

The aim of this paper is to present how neural networks can help dealing with the development of new resins, starting from the end user properties to set up the reactor's operational conditions. This procedure if followed can immensely reduce the number of experiments, pilot-plant tests, polymer characterization tests and most of all time and money spent on development. Thus, bringing real competitive advantage.

The problem with this procedure is the great amount of data that must be gathered to train the neural networks used in the procedure. The data that must be gathered account for physicochemical - end properties relationships and for the physicochemical properties of the polymer produced by different operational conditions of the reactor.

Those data can be obtained through experimental work or by means of mathematical models. Unless a great amount of experimental data is easily available, using simulated data should be considered to train the neural networks, since one of the objectives of the procedure presented wherein is to save time and money spent on experiments. Data for physicochemical - end properties relationships can be obtained by molecular modeling and group interaction techniques (Askadskii, 1987; Bicerano, 1992; VanKrevelen, 1990; Porter, 1995). Data for the polymer characteristics produced by each operational condition can be obtained by running well defined rigorous mathematical models 
for the polymer reactor. In this paper the fluidized bed reactor was used as case study, a reactor type which has two good models available: Fernandes and Lona (2001) and Hatzantonis et al. (2000).

\section{Neural Network Models}

Neural networks have been attracting great interest as predictive models, as well as for pattern recognition. The potential for using neural networks in industrial applications is enormous. Neural networks have the ability of learning the behavior of the process and the relationships between variables, without having a model of the phenomenological laws that rules the system. The success in obtaining a reliable and robust network depends strongly on the choice of process variables involved, as well as the available set of data and the domain used for training purposes (Nascimento et al., 2000).

In general, the network consists of processing neurons and information flow channels between the neurons, usually denominated interconnects. Each processing neuron calculates the weighted sum of all interconnected signals from the previous layer plus a bias term, and then generates an output through its activation sigmoid functions.

In this work, the back propagation algorithm was used, because it is the most extensively adopted algorithm and was predicted well the data presented. The available data were split in two sets for each neural network in the method. One set was used to train the network and the other to test its prediction capability. Special attention was kept in order to not overtrain the networks.

\section{Polyethylene Production in Fluidized Bed Reactors}

The fluidized bed reactor for polyethylene production comprises three different phases (bubble, emulsion gas and particulate). Reacting and inert gases are fed into the bottom of the reactor through a distributor and splits to form the bubble and emulsion gas phases. The gas in excess for maintaining the minimum fluidization condition passes through the bed as bubbles. Unreacted gases exiting from the top of the fluidized bed of polymer flows upwardly through a disengaging zone in the upper part of the reactor. The disengaging zone normally is larger in diameter than the polymerization zone, so as to reduce the gas flow velocity and thereby facilitate the settling out of solid particulate. Particles that pass the disengaging

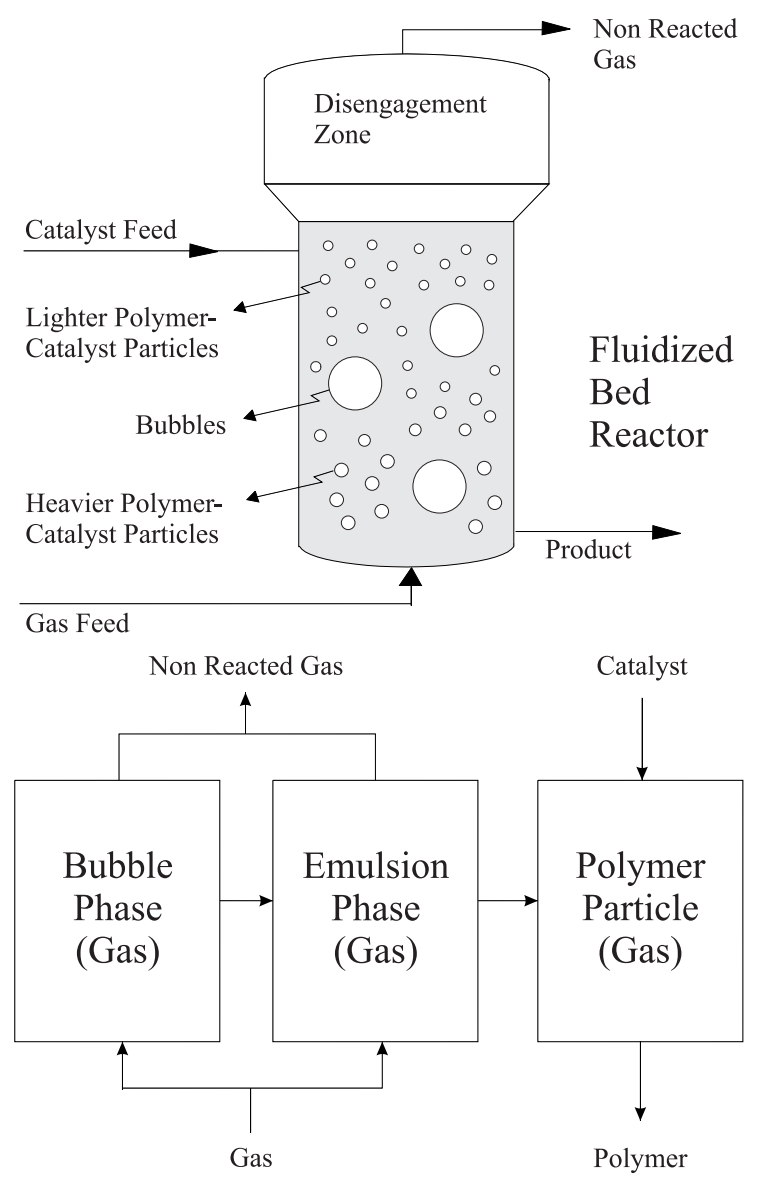

Figure 1. Fluidized bed polymerization reactor and phases diagram.

zone are separated from the gas phase by means of a cyclone and then are returned to the fluidized bed.

The catalyst is generally fed near the top of the reactor. As reaction proceeds, polymer is formed in the catalyst surface. During operation of the reactor, new polymer product is continuously formed by the catalyzed polymerization of the gas and product is continuously withdrawn to maintain the fluidized polymer product bed at a constant level.

Figure 1 shows a schematic view of the fluidized bed reactor and a diagram of the phases inside the reactor.

Data

\section{Polymer End Properties}

Great part of the polymer properties are dependent on its structure, especially on the molecular weight and the monomer units that build the polymer structure. Some variables can be temperature, time or pressure dependent.

A large quantity of polymer properties (more than 150) can be estimated from group interaction 
modeling, correlations and physical laws. These calculation methods were extensively reviewed by VanKrevelen (1990) and Porter (1995).

The properties used to train the neural networks used to develop this method were obtained by estimating 120 different end properties for polyethylene-1-butene copolymer with different molecular weight and monomer fractions in the polymer structure. The software PolyPPS was used to generate the data used to train and test the neural networks.

\section{Fluidized Bed Reactor Operational Conditions and Basic Properties}

The model developed by Fernandes and Lona $(1999,2001)$ for fluidized bed, and used in this work, is a three phase heterogeneous model. This model differs from the two phase model (bubble and emulsion) by splitting the emulsion phase in two different phases: emulsion gas phase and particulate phase (polymer particles). This division allows a more detailed modeling of the polymer, since during production the study of its development, morphology and characterization is extremely important. The model was developed focusing on the polymer particles, being the polymerization reaction and polymer development key elements for the resolution of the model.

The equations that rule the model are based on the polymerization time rather than on the reactor height (as dome by traditional models). It is assumed that the particles have a plug-flow behavior flowing from the top of the reactor in direction to the base where the polymer is removed from the reactor.

Bubble and gas emulsion phases are related to the particulate phase through the time contact ratio between the phases. The prediction of the reactor behavior throughout the reactor height is obtained by converting polymerization time into reactor position using the velocity of each phase.

Mass and energy balances for the particulate phase is given by Equations 1 and 2 .

$$
\begin{aligned}
& \frac{\partial C_{p}}{\partial t}=\frac{1}{r_{p}^{2}} \frac{\partial}{\partial r_{p}}\left(D_{p} \cdot r_{p}^{2} \frac{\partial C_{p}}{\partial r_{p}}\right)-R p \\
& \frac{\partial T_{p}}{\partial t}=\frac{1}{r_{p}^{2}} \frac{\partial}{\partial r_{p}}\left(\left(\frac{k_{e}}{\rho_{p} \cdot C p_{p}}\right) \cdot r_{p}^{2} \frac{\partial T_{p}}{\partial r_{p}}\right)+\frac{(-\Delta H)}{\rho_{p} \cdot C p_{p}} R p
\end{aligned}
$$

The material balance for the emulsion gas phase assumes the consumption of the gases during polymerization reaction and the mass transfer between bubble and emulsion phases (Equation 3). Energy balance for the emulsion phase assumes the energy transferred by diffusion due to temperature gradient between bubble and emulsion and particle and emulsion phase and also to the energy transferred due to mass transfer (Equation 4). Energy and material balance for the bubble phase is given by the equations 5 and 6.

$$
\begin{aligned}
& \frac{d C_{e}}{d t}=R p \cdot \frac{U_{p} \cdot \mathcal{E}}{U_{e} \cdot(1-\varepsilon)}-\frac{K m \cdot\left(C_{e}-C_{b}\right) \cdot U_{p} \cdot \delta}{U_{b} \cdot(1-\delta) \cdot \varepsilon} \\
& \frac{d T_{e}}{d t}=-\frac{h_{p} \cdot\left(T_{p}-T_{e}\right)}{\left(C p_{e} \cdot C_{e}\right)}+\frac{\left[H_{m}+C p_{b} \cdot K m \cdot\left(C_{b}-C_{e}\right)\right]\left(T_{e}-T_{b}\right)}{\left(C p_{b} \cdot C_{b}\right)} \\
& \frac{d C_{b}}{d t}=-\frac{K m \cdot\left(C_{e}-C_{b}\right) \cdot U_{p} \cdot \delta}{U_{b} \cdot(1-\delta) \cdot \varepsilon} \\
& \frac{d T_{b}}{d t}=\frac{\left[H_{m}+C p_{b} \cdot K m \cdot\left(C_{b}-C_{e}\right)\right] \cdot\left(T_{e}-T_{b}\right)}{\left(C p_{b} \cdot C_{b}\right)}
\end{aligned}
$$

The polymer properties characterization is simulated using the method of moments (Zabisky et al., 1992). Application of this method allows the prediction of several physicochemical characteristics of the polymer (molecular weights, density, polydispersity, melt index and active sites occupation and efficiency). The reaction mechanism used in this work is presented in Table 1.

The polymerization rate is given by equation 7 , Table 1. Reaction mechanism for ethylene - 1-butene copolymerization through coordination reaction using Ziegler-Natta catalyst (McAuley et al., 1990).

\begin{tabular}{ll}
\hline Formation & $R^{*} \stackrel{k f}{\longrightarrow} R_{0}$ \\
\hline Initiation & $R_{0}+C_{i} \stackrel{k i}{\longrightarrow} R_{i}(l)$ \\
& $H^{*}+C_{i} \stackrel{k h i}{\longrightarrow} R_{i}(l)$ \\
& $H^{*}+X \stackrel{k h r}{\longrightarrow} R_{X}(1)$ \\
\hline Propagation & $R_{i}(r)+C_{k} \stackrel{k p i k}{\longrightarrow} R_{k}(r+l)$ \\
\hline Chain Transfer & \\
to monomer & $R_{i}(r)+C_{k} \stackrel{k f m i k}{\longrightarrow} P(r)+R_{k}(l)$ \\
to hydrogen & $R_{i}(r)+H_{2} \stackrel{k f h i}{\longrightarrow} P(r)+H^{*}$ \\
to cocatalyst & $R_{i}(r)+X \stackrel{k f r i}{\longrightarrow} R_{X}(1)+P(r)$ \\
spontaneous & $R_{i}(r) \stackrel{k f s i}{\longrightarrow} P(r)+H^{*}$ \\
\hline
\end{tabular}


depending mainly on the propagation rate, monomer concentration and the zero-moment of the live polymer. Copolymerization, terminal effects and active site type are also considered.

$$
R p=\sum_{j} \sum_{i} \sum_{k} k p_{i k} \cdot M_{k} \cdot Y_{0}(j)
$$

The simulations with the model have been outputting the expected results for industrial fluidized bed reactors. Test with the model were done with literature and patent data (Brule et al., 1993; Goeke et al., 1983; Jenkins et al., 1985; McAuley et al, 1994). Validation of the model was done based on information such as concentration profiles, consumption of reagents, productivity and polymer characteristics.

\section{Development of New Polymer Grades using Neural Networks}

To predict the operational conditions of the fluidized bed reactor that will produce the polymer with the clients desired end properties it is necessary first to determine the basic physicochemical properties of the polymer (molecular weight and chain structure) from the end property and then to determine the operational conditions from the basic physicochemical properties.

If the scheme of Figure 2 is run up to the first part, it will be predicting the basic polymer properties and this procedure can be used to select the most probable commercial resin that the client should get. If the scheme

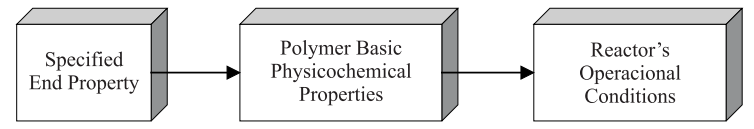

Figure 2. Prediction Flowchart.

is run to the second stage (prediction of the operational conditions) this procedure can be used for polymer grade development. The entire neural network configurations used in this procedure is shown in figure 3.

\section{Prediction of the Basic Physicochemical Properties}

As said before, the first part consists in determining the basic physicochemical properties of the polymer from the end user properties. This prediction is done by network 1 . Since polymer properties are directly related to its molecular structure it is not possible to specify too many properties for the polymer, because by specifying some properties other properties will be automatically being specified. This is because they are inherent to the same polymer chain structure and molecular weight.

In general, only two end properties can be chosen to specify a polymer grade. A list of the most common end properties that are required by the plastic industry is shown in Table 2.

For example: values for density and viscosity can be specified since density is a function of the polymer composition and viscosity is a function of composition and molecular weight. On the other hand,

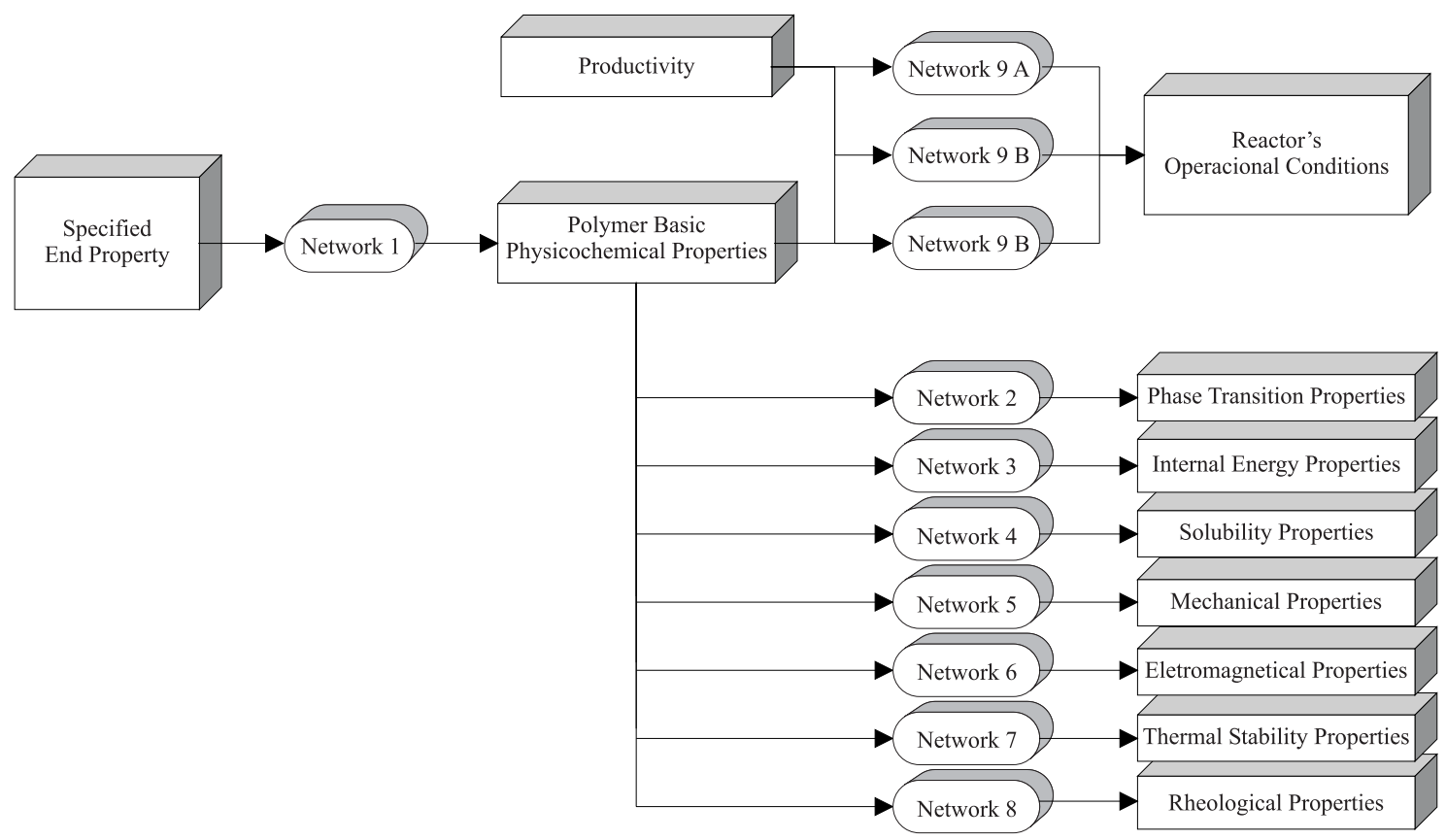

Figure 3. Complete neural network flowchart 
Table 2. Most common end properties specified by the plastic industry.
Melting Temperature
Opacity
Impact Resistance
Strain Stress Tension
Hardness
Mechanical Modules
Density
Viscosity

values for density and melting temperature could not be specified, since both are functions of the polymer composition and by setting one of them, the other will be automatically set. So to have the knowledge of what influences each end user property is very important.

Network 1 is really a series of networks. Since there are $\mathrm{x}$ end properties that can be specified, then $\mathrm{x}$ neural networks have to exist in order to output the basic polymer properties with the specified property. When two end properties are specified then stacked neural networks (Zhang et al., 1997) are used in order to combine the results of the individual network outputs.

For the polyethylene copolymer case, network 1 needs only one hidden layer with 8 neurons to output predictions with errors of 0.01 to $0.1 \%$ what can be considered an excellent prediction. Training of the individual networks (one for each end property) is very fast and only a few data points are needed (ten data points where used for polyethylene copolymers). Errors were calculated as:

$$
e=\left|\frac{(\text { simulation data })-(\text { NN prediction })}{(\text { simulation data })}\right| \cdot 100[\%]
$$

With the predictions of the basic polymer characteristics it is possible to use the networks 2 to 8 to predict the other polymer properties related to the polymer chain structure found by network 1 . Network 2 to 8 are trained with the data collected with the PolyPPS software used to predict the polymer properties. Again, a neural network topology of one hidden layer and 8 neurons in this layer was enough to predict the other end properties with an error lower than $0.1 \%$. Training of these networks is also fast. Alternatively, a predestine algorithm that uses group interaction to estimate polymer end properties can also be used at this point.

\section{Prediction of the Reactor's Operational Conditions}

The biggest challenge of this procedure is to train network 9 which will predict the operational condition of the fluidized bed reactor based on the basic polymer properties and the desired productivity.

Fluidized bed reactors, due to its complexity, has at least 6 variables that need to be specified as operational conditions: concentrations at feed, temperature at feed, catalyst flow rate, superficial velocity, bed porosity and pressure. Network 9 must provide all these information.

The data used to train this network was obtained running 176 simulations using Fernandes and Lona (2001) fluidized bed model. The simulations were chosen carefully using experimental design technique in order to cover a full $3^{\mathrm{n}}$ factorial design with the possible operational conditions that can be employed in the reactor under industrial conditions. Table 3 presents the range of the operational conditions used for each variable.

The first attempts for the topology of this network was to have 2 or 3 hidden layers with at least 12 neurons and a maximum of 50 neurons in each layer. Training for these conditions have failed to output good results and training time was also very high taking more than 48 hours for some cases. Errors of prediction were greater than $20 \%$ for some test data points (Figure 4).

The error presented were due to two major factors: an unsuitable variable output and a variable difficult to train. During the test with the neural networks, big prediction problems were detected with the variables: superficial velocity and catalyst feed rate.

In fluidized bed reactors, the superficial velocity of the gas is usually expressed as the number of time it is bigger than the minumum fluidizing velocity $\left(\mathrm{U}_{0}=X . \mathrm{U}_{\mathrm{mf}}\right)$. First attempts to train the neural networks were done using this expression and the

Table 3. Range used for each operational condition variable

\begin{tabular}{lc}
\hline \multicolumn{1}{c}{ Variable } & Range \\
\hline Ethylene Concentration $(\%)$ & 35 to 50 \\
Inert Concentration $(\%)$ & 30 to 50 \\
Feed Temperature $(\mathrm{K})$ & 310 to 350 \\
Catalyst Feed Rate $(\mathrm{g} / \mathrm{s})$ & 0.05 to 0.50 \\
Superficial Velocity $\left[\mathrm{x} \mathrm{U}_{\mathrm{mf}}\right]$ & 3 to 6 \\
Porosity & 0.4 to 0.7 \\
Pressure $[\mathrm{atm}]$ & 20 to 30 \\
\hline
\end{tabular}




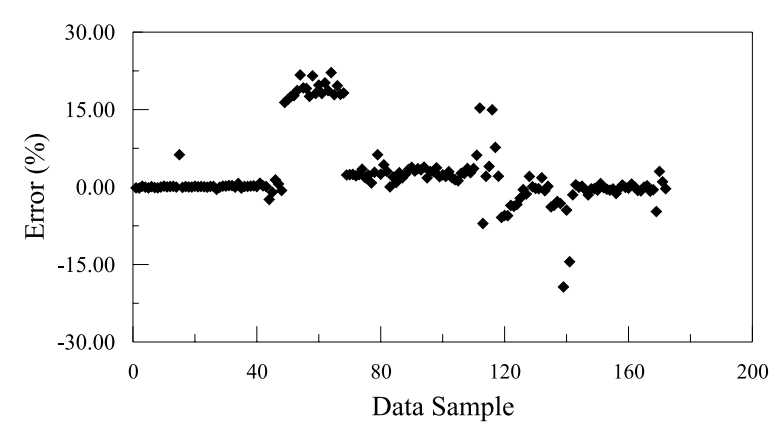

Figure 4. Error predictions for training and testing samples. Prediction of the superficial velocity (worst case). Last 35 points refer to testing data. NN topology: 2 hidden layers (30:20 neurons).

value $X$ was expected as one of the outputs of the neural network. This way of expressing the superficial velocity was proven to be unsuitable, returning very bad predictions. When training was done with the actual value of the superficial velocity (in $\mathrm{m} / \mathrm{s}$ ) the prediction error decreased. So it is important when dealing with neural networks to test the best form of training for each variable.

Even though, the best strategy encountered was training three different networks that would work with different ranges of superficial velocities (ranges: 3 to 4,4 to 5 and 5 to 6 times the minimum fluidizing velocity). The data was sorted and then used in training. Some of the data were presented to more than one network. The best topology encountered was with 3 hidden layers (20, 15 and 15 neurons). The use of the networks to predict the reactor's operational conditions was done presenting the input data (polymer physicochemical data and required productivity) to all three networks. Each network outputted an operational condition set. The best set was selected based on the quantity of catalyst used, since a lower flow rate of catalyst means a better use of the catalyst productivity potential and lower operational cost.

The second difficulty noticed was upon the catalyst feed rate. Predictions at low feed rates were very poor (predictions errors up to $30 \%$ ), whereas the predictions at high feed rates were better not surpassing $5 \%$. Since the catalyst feed rate is a critical variable for the process, affecting both production rate and molecular weight, the neural network prediction error for this variable should not be greater than $2 \%$, otherwise, the neural network application would not be useful for the estimation process. Predictions got a lot better when two more levels were added for this variable in the factorial design. Using 5 levels of values for the catalyst feed rate on the factorial design
Table 4. Example of neural network prediction for fluidized bed reactor conditions.

\begin{tabular}{l} 
Desired Polymer \\
\hline $\mathrm{Mw}=110900 \mathrm{~g} / \mathrm{mol}$ \\
Polydispersity $=3.4$ \\
$24 \%$ of 1 -Butene \\
Production $=7.5 \mathrm{ton} / \mathrm{h}$ \\
\hline
\end{tabular}

\section{Simulated Operational Conditions}

Ethylene Conc. $=0.3436 \mathrm{~mol} / \mathrm{L}$

1-Butene Conc. $=0.1475 \mathrm{~mol} / \mathrm{L}$

Superficial Velocity $=0.29 \mathrm{~m} / \mathrm{s}$

Catalyst Feed Rate $=0.075 \mathrm{~g} / \mathrm{s}$

Porosity $=0.4$

Predictions of the Neural Network

Ethylene Conc. $=0.3429 \mathrm{~mol} / \mathrm{L}$

1-Butene Conc. $=0.1479 \mathrm{~mol} / \mathrm{L}$

Superficial Velocity $=0.289 \mathrm{~m} / \mathrm{s}$

Catalyst Feed Rate $=0.0736 \mathrm{~g} / \mathrm{s}$

Porosity $=0.404$

has improved the predictions of the neural network, and a maximum error of $2 \%$ was obtained. Therefore if a variable presents a bigger error at one end of its range then the data presented to train the neural network will need at least one more level of values near the point were the predictions are bad, so the neural network will be better trained at that region.

The operational condition predicted by the networks is able to produce the polymer with the desired end property with an error lower than $5 \%$ what can be considered a good estimation (simulation results). An example of prediction is given in Table 4.

It is important to notice that the prediction may not be error free so experimentation and pilot plant test may be needed in order to tune these operational conditions, but the neural network will give a very good head start for a reduced number of experiments.

\section{Conclusions}

This work has presented a novel procedure that can be used for estimating the operational conditions of a fluidized bed reactor used to produce a polymer with some client specified end property. 
Predictions have been good with this procedure outputting estimations with less than $5 \%$ of error. For error free estimation pilot plant experimentation may be necessary and the conditions predicted by the procedure can be used as a very good head start for experimentation.

The procedure can also be used to estimate operational conditions for other kinds of reactors and for polymer selection. If used with other kinds of reactors, then the neural networks should be trained with data for that specific reactor.

\section{Acknowledgement}

FAPESP - Fundação de Amparo à Pesquisa do Estado de São Paulo.

\section{Nomenclature}

$\begin{array}{ll}\mathrm{C} & \text { concentration } \\ \mathrm{Cp} & \text { heat capacity } \\ \mathrm{D} & \text { diffusion coefficient } \\ \mathrm{h}_{\mathrm{p}} & \text { particle-emulsion heat transfer coefficient } \\ \mathrm{Hm} & \text { bubble-emulsion heat transfer coefficient } \\ \mathrm{Km} & \text { bubble-emulsion mass transfer coefficient } \\ \mathrm{M} & \text { monomer concentration } \\ \mathrm{M}_{\mathrm{W}} & \text { molecular weight } \\ \mathrm{P} & \text { dead polymer chain } \\ \mathrm{R}_{\mathrm{i}} & \text { live polymer chain with terminal monomer } i \\ \mathrm{Rp} & \text { reaction rate } \\ \mathrm{r} & \text { polymer chain length } \\ \mathrm{r}_{\mathrm{p}} & \text { polymer radius } \\ \mathrm{t} & \text { time } \\ \mathrm{T} & \text { temperature } \\ \mathrm{U} & \text { velocity } \\ \mathrm{X} & \text { cocatalyst concentration } \\ \mathrm{Y}_{0} & \text { zero-moment of the live polymer } \\ \delta & \text { void fraction } \\ \Delta \mathrm{H} & \text { heat of reaction } \\ \varepsilon & \text { porosity } \\ \rho & \text { density }\end{array}$

\section{subscripts}

$\begin{array}{ll}\mathrm{b} & \text { bubble phase } \\ \mathrm{e} & \text { emulsion phase } \\ \mathrm{p} & \text { polymer phase }\end{array}$

\section{References}

1. Askadskii, A. A. - Chemical Yearbook IV. Eds. R.A.Pethrick \& G.E. Zaikov. Harwood Acad. Publ., London (1987).
2. Bicerano, J. - Computational Modeling of Polymers. Marcel Dekker, New York (1992).

3. Brule, D.; Iffly, J. M. \& Le, J. - Process and device for the gas phase polymerization of alpha-olefins. US.Patent 5241023 (1993).

4. Fernandes, F. A. N. \& Lona, L. M. F. - Fluidized bed reactor and physical-chemical properties modeling for polyethylene production. Computers and Chemical Engineering, 23, S803-S806 (1999).

5. Fernandes, F.A.N. \& Lona, L.M.F. - Heterogeneous modeling for fluidized bed polymerization reactor. Chemical Engineering Science, 56, 963-969 (2001).

6. Goeke, G. L.; Wagner, B. E. \& Karol, F. J. - Process for the preparation of high density ethylene polymers in fluid bed reactor. US.Patent 4383095 (1983).

7. Hatzantonis, H., Yiannoulakis, H., Yiagopoulos, A. \& Kiparissides, C. - Recent developments in modeling gas-phase catalyzed olefin polymerization fluidized-bed reactors: The effect of bubble size variation on the reactor's performance. Chemical Engineering Science, 55, 3237-3259 (2000).

8. Jenkins III, J. M.; Jones, R. L. \& Jones, T. M. - Fluidized bed reaction systems. US.Patent 4543399 (1985).

9. McAuley, K. B., MacGregor, J. F. \& Hamielec, A.E. - A kinetic model for industrial gas-phase ethylene copolymerization. AIChE Journal, 36, 837-850 (1990).

10. McAuley, K. B., Talbot, J. P. \& Harris, T. J. - A comparison of two-phase and well-mixed models for fluidized-bed polyethylene reactors. Chemical Engineering Science, 49, 2035-2045 (1994).

11. Nascimento, C. A. O.; Giudici, R. \& Guardani, R. - Neural network based approach for optimization of industrial chemical processes. Computers and Chemical Engineering, 24, 2303-2314 (2000).

12. Porter, D. - Interaction Modelling of Polymer Properties. Marcel Dekker, New York (1995).

13. Van Krevelen, D.W. - Properties of Polymers. Elsevier, New York (1990).

14. Zhang, J.; Martin, E. B.; Morris, A. J. \& Kiparissides, C. - Inferential estimation of polymer quality using stacked neural networks. Computers and Chemical Engineering, 21, S1025-S1030 (1997).

15. Zabisky, R. C. M.; Chan, W. M.; Gloor, P. E. \& Hamielec, A. E. - A kinetic model for olefin polymerization in high-pressure tubular reactors: a review and update. Polymer, 33, 2243-2262 (1992).

Recebido: 10/08/01

Aprovado: 06/04/02 